OPEN ACCESS

Edited by:

Elisa Alos,

Pompeu Fabra University, Spain

Reviewed by:

Edward Tower

Harvard University, United States Dessislava Pachamanova

Babson College, United States

${ }^{*}$ Correspondence: Josselin Garnier josselin.garnier@polytechnique.edu

Specialty section

This article was submitted to Mathematical Finance,

a section of the journal

Frontiers in Applied Mathematics and

Statistics

Received: 30 December 2019 Accepted: 26 March 2020

Published: 29 April 2020

Citation:

Garnier J and Sølna K (2020) Implied Volatility Structure in Turbulent and Long-Memory Markets.

Front. Appl. Math. Stat. 6:10. doi: 10.3389/fams.2020.00010

\section{Implied Volatility Structure in Turbulent and Long-Memory Markets}

\author{
Josselin Garnier ${ }^{1 *}$ and Knut Sølna ${ }^{2}$ \\ ${ }^{1}$ CMAP, CNRS, Ecole Polytechnique, Institut Polytechnique de Paris, Palaiseau, France, ${ }^{2}$ Department of Mathematics, \\ University of California, Irvine, Irvine, CA, United States
}

We consider fractional stochastic volatility models that extend the classic Black-Scholes model for asset prices. The models are general and motivated by recent empirical results regarding the behavior of realized volatility. While such models retain the semimartingale property for the asset price the associated European option pricing problem becomes complex, with no explicit solution. In a number of canonical scaling regimes it is possible, however, to derive asymptotic and sparse representations for the option price and the associated implied volatility, that are parameterized by a few effective parameters and that involve power law dependencies on time to maturity. These effective parameters may depend in a complicated way on the volatility model, but they can be easily estimated from the observation of a few option prices. The effective parameters associated with a particular underlying asset can be calibrated with respect to liquid contracts written on this asset and then used for pricing less liquid contracts written on the same underlying asset. Therefore, the effective parameters provide a robust link between financial products written on a particular underlying asset.

Keywords: stochastic volatility, long-memory process, fractional process, volterra process, asymptotics, time scales, option price, implied volatility

\section{INTRODUCTION}

In the classic Black-Scholes modeling framework the asset price has independent Gaussian returns and a constant volatility (see [1]). However, European option prices predicted by this idealized model often do not capture well market-traded option prices. The discrepancy is typically parameterized by the implied volatility, that is, the volatility that needs to be used in the BlackScholes pricing formula to replicate the market-traded option prices. The implied volatility is then a function of the contract parameters, the maturity, and the strike in the case of European options, while in the ideal Black-Scholes situation the implied volatility should be constant. In fact, the implied volatility calibrated from past option pricing data can be seen as a proxy for prices and used in the pricing of tomorrow's options. This is in contrast to an approach where calibration of the underlying asset model, in particular its volatility, is used to price options.

In order to carry out a robust calibration it is desirable to have a good model for the implied volatility, a model that somehow captures the essence of how the market drives and modulates the option prices. Stochastic volatility models have been very successful in explaining the behavior of the implied volatility. Here the volatility of the underlying asset price process is modeled as a stochastic process itself, which leads to a particular form for the implied volatility. For background on stochastic volatility models we refer to the books and surveys [1-6], and the references therein. The form of the implied volatility is explicit only for some special classes of stochastic volatility models, in particular the Heston [7] model. It has been observed that the Heston [7] model does 
not always fit the observations well. Therefore, other models have been introduced in the literature, models where there is no explicit expression for the implied volatility. That is why it is of interest to use asymptotic techniques to get explicit expressions that approximate the implied volatility in specific parameter regimes as explained by Fouque et al. [1]. The main idea is to identify a set of canonical scaling regimes where asymptotic techniques can be used to determine accurate option price approximations giving sparsely parameterized models for the implied volatility. The estimation of the parameters from market-traded option prices is then possible and robust. Most of the literature on stochastic volatility has focussed on situations when the volatility process is a Markov process, typically some sort of a jump diffusion process. However, a number of empirical studies such as Gatheral et al. [8] suggest that the volatility process possesses power-law correlations that decay relatively slowly compared to the exponential rate of Markov models. Here we develop a general framework for modeling of nonMarkov processes and use it in the context of stochastic volatility. This general framework for modeling of non-Markov stochastic processes is based on a fractional Volterra process formulation. The distribution of the future evolution of such a non-Markov process cannot be described knowing only the present value of the process, the past history of the process is also needed and we say that the process has memory.

The main asymptotic regimes considered here are the ones where (1) the standard deviation of the stationary fluctuations of the stochastic volatility is of the same order as the average, (2) the time to maturity is of the same order as the diffusion time, and (3) the mean reversion time of the stochastic volatility is either shorter or longer than the diffusion time. Thus, we address both the rapid and slow mean reversion regimes in this paper. Here the diffusion time refers to the natural evolution time scale of the asset price (it can be defined as the reciprocal of the square effective volatility). In these regimes the leadingorder expression of the implied volatility surface is linear in log-moneyness, with moneyness being the option strike relative to the current asset value. This may seem somewhat restrictive from the point of view of fitting since a strong skew in logmoneyness can be observed in certain markets. This has been observed for the stock market, but less so for other markets like fixed income markets. However, if one considers higherorder approximations, then our framework generates strong skew effects. A number of other modeling issues like transaction costs, bid-ask spreads, interest rate, market price of risk, and liquidity issues may also affect the skew shape, but are not considered here. The fractional volatility model we set forth here incorporates empirical "stylized facts," like heavy tails of returns, volatility clustering, and mean reversion, moreover, volatility persistence as observed from empirical viewpoints by Cont [9]. Additionally, we incorporate the leverage effect, which is a term coined by Black [10] referring to stock price movements which are correlated (typically negatively) with volatility, as falling stock prices may imply more uncertainty and hence more volatility. We seek here to answer the question about what parametric forms for the implied volatility long- and short-range stochastic volatility fluctuations produce and we use asymptotic techniques to do so. Here short-range volatility refers to situations when the volatility path is rougher than in the Markovian case while longrange refers to situations when the volatility is smoother than in the Markovian case. Such a description is useful in the context of calibration and linkage of financial contracts written on the same underlying asset via calibration of liquid contracts with respect to implied volatility. Here, we consider just one piece of this challenge which involves characterization of the implied volatility surface for long- and short-range stochastic volatilities. An underlying premiss is that constructing a parametric form for the implied volatility that derives from a stochastic volatility that is a stationary process helps in designing a scheme that is time consistent and robust. Moreover, it provides the appropriate degrees of freedom and a framework for linkage of financial products. Long- and short-range stochastic volatility models are indeed easy to pose, however, their analysis is quite challenging. This comes from the fact that the volatility process is then neither a Markov process nor a semimartingale. The price process is still, however, a semimartingale and the problem formulation does not entail arbitrage as shown by Mendes et al. [11].

We consider a general framework for modeling with shortand long-range fractional processes and application of this to asymptotic option pricing. This builds on the work by Garnier and Sølna [12-14] by extending the results from fractional Ornstein-Uhlenbeck processes to general Volterra processes. Such frameworks were also used in the context of optimal portfolio construction by Fouque and $\mathrm{Hu}$ [15-17] and in hedging by Garnier and Sølna [18]. In these papers the time to maturity is of the same order as the characteristic diffusion time. The case of small volatility asymptotics in the stationary case is discussed in Garnier and Sølna [12] while here the focus is on asymptotics associated with separation of time scales.

The outline of the paper is as follows. We start with a review of some relevant literature in section 2. Then, in section 3 we introduce the fractional Volterra stochastic volatility model and discuss the properties of the autocovariance of this model for three classes $(\mathrm{St}),(\mathrm{RoH})$, and $(\mathrm{LoH})$. Then we introduce the three important time scales, the volatility mean reversion time, the diffusion time, and the time to maturity in section 4 . We exploit separation of these time scales to derive asymptotic expressions for respectively option prices and implied volatility in sections 5 , 6. We discuss some calibration issues in section 7 and we finish with some conclusions in section 8 .

\section{LITERATURE REVIEW ON NON-MARKOV STOCHASTIC VOLATILITY MODELS}

Most of the literature on stochastic volatility has focussed on situations when the volatility process is a Markov process, typically some sort of a jump diffusion process. Based on the observed structures of the returns and of the implied volatility surface $[19,20]$ claim that the asset price should be a jump process. Models with asset price jumps and stochastic volatility models both lead to heavy returns and have been used to capture smile dynamics. Such behaviors are also produced by models 
driven by general Lévy processes, both for volatility models as in Figueroa [21] and Mijatovic and Tankov [22] as well as for the asset price itself as in Barndorff-Nielsen et al. [23]. A number of recent empirical studies suggest, however, that the volatility process possesses long- and/or short-range dependence and is non-Markovian. Stochastic volatility models with memory have recently received a lot of attention and this work is motivated in part by the analysis of volatility of historical prices and in part by the structure of the implied volatility as reported by Breidt et al. [24], Charfeddine [25], Chronopoulou and Viens [26], Gatheral et al. [8], and Oh et al. [27] for instance.

The classic model for processes with memory is the fractional Brownian motion ( $\mathrm{fBm}$ ) introduced by Mandelbrot and Van Ness [28]. This is a one-parameter generalization of the standard Brownian motion. The parameter characterizing this process is the Hurst index $H \in(0,1)$. The $\mathrm{fBm}$ process has paths that are almost surely Hölder continuous with exponent $\gamma$ for any $\gamma<H$. Two qualitatively different cases can be identified. The case with $H<1 / 2$ corresponds to a process whose successive increments are negatively correlated. Such a process is referred to as an anti-persistent, rough, or fractional short-range process. The process is then indeed rougher than standard Brownian motion. The case with $H>1 / 2$ gives positively correlated increments. Such a process is referred to as a persistent or fractional long-range process. The correlations of the increments of the $\mathrm{fBm}$ decay as $\Delta t^{2 H-2}$ as a function of the separation $\Delta t$ between the increments, so that the autocorrelation function of the increment process is not integrable in the long-range case. This is in contrast to the case with a Markov process whose correlations decay at an exponential rate. We will see below that the persistent and anti-persistent cases give qualitatively different structures for the implied volatility. Note that the case $H=$ $1 / 2$ corresponds to standard Brownian motion, whose successive increments are independent, and gives a Markov process. Here we use a general short- or long-range Volterra class of models which is driven by standard Brownian motion. The stochastic volatility itself is modeled as a smooth function of the Volterra process. Indeed this process will be stationary in a strong sense with the distribution of the process being invariant with respect to time shifts. As a special case we discuss a fractional OrnsteinUhlenbeck process driven by fractional Brownian motion on $\mathbb{R}$ of the type introduced by Mandelbrot and Van Ness [28] (type I $\mathrm{fBm}$ ). Fractional Brownian motion on $\mathbb{R}^{+}$can also be constructed via a moving average representation of Brownian motion on $\mathbb{R}^{+}$ (type II fBm) as described for instance in Biagini et al. [29]. When modeling the volatility in the financial context it is important to use a driving process whose increments are "memory-stationary" and that is why we use the $\mathrm{fBm}$ representation of Mandelbrot and Van Ness [28].

There has been strong interest in modeling and analysis of fractional stochastic volatility in recent years. Comte et al. [30] consider a long memory extension of the Heston [7] option pricing model, a fractionally integrated square root process that is a generalization of the early work by Comte and Renault [31]. Further results on the fractional Heston model are found for instance by El Euch and Rosenbaum [32] and Guennoun et al. [33], where short- and long-time to maturity asymptotics are studied using large deviations principles. In the Markov case the integrated square volatility would converge to its mean value exponentially fast and this would flatten the implied volatility term structure. In Comte et al. [30], it is discussed how long-range dependence provides an explanation for observations of non-flat term structure in the regime of long time to maturity since the long-range dependence can make the implied volatility smile strongly maturity-dependent and can also produce consistent smiles for short time to maturity. It may be argued that the typical case for stochastic volatility is the fractional rough case with $H<1 / 2$, which is the point of view set forth in Gatheral et al. [8]. However, empirical findings of long-range dependence structure have also been reported, for instance in Chronopoulou and Viens [26]. Long-range volatility situations have been reported for currencies in Walther et al. [34], for commodities in Charfeddine [25] and for equity index in Chia et al. [35], while analysis of electricity markets data typically gives $H<1 / 2$ as in Bennedsen [36], Rypdal and Lovsletten [37], and Simonsen [38]. Both the short- and long-range fractional behaviors can be observed depending on the specific market and regime and they are, therefore, important.

In the previous articles the time to maturity is of the same order as the characteristic diffusion time (the reciprocal of the square effective volatility). A large number of recent papers consider asymptotic approximations in the regime of short time to maturity. In Alòs et al. [39], the authors use Malliavin calculus to decompose option prices as the sum of the classical BlackScholes formula with volatility parameter equal to the rootmean-square average of the future volatility plus a term due to correlation and a term due to the volatility of the volatility. Their model is a fractional version of the Bates [40] model. They find that the implied volatility flattens in the long-range dependent case in the limit of short time to maturity. We make a connection to this result below in section 6.1. Forde and Zhang [41] use large deviation theory to determine the short time to maturity asymptotic form of the implied volatility. They consider the correlated case with leverage and obtain results that are consistent with those in Alòs et al. [39]. They consider stochastic volatility models based on $\mathrm{fBms}$ which are analyzed by rough path theory. They also consider long time to maturity asymptotics for some fractional processes. Small time to maturity asymptotic results are presented by Chronopoulou and Viens [42] and Gulisashvili [43]. Another important asymptotic regime corresponds to small volatility fluctuations. Fukasawa [44] considers this situation and discusses the impact of longrange dependence on the implied volatility. He uses a nonstationary $\mathrm{fBm}$ as the volatility factor so that the leading implied volatility surface is identified conditioned on the present value of the implied volatility factor only. Here we consider a stationary model so that the surface depends in general on the path of the volatility factor until the present, reflecting the non-Markovian nature of $\mathrm{fBm}$. The case of small volatility asymptotics in the stationary case is discussed in Garnier and Sølna [12] while here the focus is on asymptotics associated with separation of time scales.

We remark finally that a further generalization, relative to $\mathrm{fBm}$ based models, is the case of multi-fractional Brownian motion 
based models (see for instance [45]). This makes it possible to consider a non-stationary local regularity and a time-dependent Hurst index, so that the implied volatility depends on weighted averages of the local Hurst index. Here we consider a monofractal situation with a fixed value for the Hurst index, the multi-fractal cases will be considered elsewhere.

\section{STOCHASTIC VOLATILITY MODEL}

The price $X_{t}$ of the risky asset follows, under the pricing measure, the stochastic differential equation

$$
d X_{t}=\sigma_{t} X_{t} d W_{t}^{*}
$$

where the stochastic volatility is of the form

$$
\sigma_{t}=F\left(Z_{t}\right)
$$

In the standard Black-Scholes model the volatility $\sigma_{t}$ is constant while here it is a stochastic process which reflects variations in the market conditions. The function $F$ is a deterministic smooth function, called the volatility function. The stochastic process $Z_{t}$ is a Volterra process, called the volatility factor. This process produces the fluctuations in the volatility and determines its time scale contents. The stochastic volatility is adapted to the Brownian motion $W_{t}$. The Brownian motion $W_{t}^{*}$ driving the asset is correlated to the stochastic volatility through

$$
W_{t}^{*}=\rho W_{t}+\sqrt{1-\rho^{2}} B_{t}
$$

where the Brownian motion $B_{t}$ is independent of $W_{t}$ and $\rho \in[-1,1]$. In the standard Black-Scholes framework there are explicit expressions for vanilla option prices like European call option prices. In the general stochastic volatility case there are no such explicit expressions. However, in certain regimes, characterized by the relative magnitudes of the characteristic time scales of the price process $X_{t}$, we can derive asymptotic formulas for the option prices and we discuss such asymptotics in this paper when the volatility factor is a fractional Volterra process.

\subsection{The Volatility Factor}

We assume that the volatility factor has the form

$$
Z_{t}=\frac{\sigma_{Z}}{\sqrt{\tau_{Z}}} \int_{-\infty}^{t} \mathcal{K}\left(\frac{t-s}{\tau_{Z}}\right) d W_{s}
$$

where $\mathcal{K}$ is a function in $L^{2}(0, \infty)$ with $\int_{0}^{\infty} \mathcal{K}(u)^{2} d u=1$. The volatility factor is a zero-mean, stationary, Gaussian process with variance

$$
\mathbb{E}\left[Z_{t}^{2}\right]=\sigma_{Z}^{2}
$$

and covariance that is a function of $s / \tau_{Z}$ :

$$
\mathbb{E}\left[Z_{t} Z_{t+s}\right]=\sigma_{Z}^{2} \mathcal{C}_{Z}\left(\frac{s}{\tau_{Z}}\right), \quad \mathcal{C}_{Z}(s)=\int_{0}^{\infty} \mathcal{K}(u) \mathcal{K}(s+u) d u
$$

The normalized correlation function $\mathcal{C}_{Z}(s)$ is continuous, its value at zero is one and it goes to zero at infinity. Its width is of order one, so that $\tau_{Z}$ is the mean-reversion time of the volatility.

In this paper we shall consider three different classes.

- We say that the volatility factor belongs to the standard class (St) if $\mathcal{K} \in L^{1} \cap L^{\infty}$. For technical reasons we add the assumption: $\mathcal{K}$ is Lipschitz on $(0,+\infty)$. The main assumptions mean that the volatility factor has a standard behavior at infinity (i.e., its correlation function is integrable) and at zero (i.e., its correlation function is of the form $1-O(|s|)$ for small $s$ ). A typical example is a standard Ornstein-Uhlenbeck process.

- We say that the volatility factor belongs to the class $(\mathrm{RoH})$ for some $H \in(0,1 / 2)$ if $\mathcal{K} \in L^{1}$ and there exists $d_{Z} \neq 0$ such that

$$
\mathcal{K}(t)=d_{Z} t^{H-\frac{1}{2}}(1+o(1)), \quad t \rightarrow 0 .
$$

For technical reasons we add the assumption: $\mathcal{K}$ is Lipschitz on $(1,+\infty)$ and $t \mapsto \mathcal{K}(t)-d_{Z} t^{H-1 / 2}$ is $H^{\prime}$-Hölder on $(0,1)$ for some $H^{\prime}>H$. The main assumptions imply that the volatility factor has a standard behavior at infinity (i.e., its correlation function is integrable), but a rough behavior at zero (see [12]):

$$
\mathcal{C}_{Z}(s)=1-q_{Z}|s|^{2 H}+o\left(|s|^{2 H}\right) \text { as }|s| \rightarrow 0
$$

with

$$
q_{Z}=d_{Z}^{2} \frac{\Gamma\left(H+\frac{1}{2}\right)^{2}}{2 \sin (\pi H) \Gamma(2 H+1)},
$$

that is to say, the process has short-range correlation properties. The process is "rough" in that it is $H^{\prime}$ Hölder continuous for any $H^{\prime}<H$, but not for $H^{\prime}>H$. A typical example is a fractional Ornstein-Uhlenbeck process with the Hurst index $H \in(0,1 / 2)$ as explained below.

- We say that the volatility factor belongs to the class $(\mathrm{LoH})$ for some $H \in(1 / 2,1)$ if $\mathcal{K} \in L^{\infty}$ and there exists $c_{Z} \neq 0$ such that

$$
\mathcal{K}(t)=c_{Z} t^{H-\frac{3}{2}}(1+o(1)), \quad t \rightarrow+\infty .
$$

For technical reasons we add the assumption: $\mathcal{K}$ is Lipschitz on $(0,+\infty)$ and $t \mapsto \mathcal{K}(t)-c_{Z} t^{H-3 / 2}$ is in $L^{1}$. The main assumptions mean that the volatility factor has a standard behavior at zero, but its correlation function is not in $L^{1}$ (see [12]):

$$
\mathcal{C}_{Z}(s)=k_{Z}|s|^{2 H-2}(1+o(1)) \text { as }|s| \rightarrow+\infty
$$

with

$$
k_{Z}=c_{Z}^{2} \frac{\Gamma\left(H-\frac{1}{2}\right)^{2}}{2 \sin (\pi H) \Gamma(2 H-1)} .
$$

With such slowly decaying correlations we say that the process has long-range correlation properties. A typical example is a fractional Ornstein-Uhlenbeck process with the Hurst index $H \in$ $(1 / 2,1)$ as discussed below. 


\subsection{The Volatility Function}

The volatility function $F$ is assumed to be one-to-one, positivevalued, smooth, bounded, and with bounded derivatives. Accordingly, the filtration $\mathcal{F}_{t}$ generated by $\left(B_{t}, W_{t}\right)$ is the one generated by $X_{t}$. Indeed [see [14]], it is equivalent to the filtration generated by $\left(W_{t}^{*}, W_{t}\right)$, or $\left(W_{t}^{*}, Z_{t}\right)$. Because $F$ is one-to-one, it is equivalent to the filtration generated by $\left(W_{t}^{*}, \sigma_{t}\right)$. Because $F$ is positive-valued, it is equivalent to the filtration generated by $\left(W_{t}^{*}, \sigma_{t}^{2}\right)$, or $X_{t}$.

In order to establish some results in the $(L o H)$ case, we need to assume additional conditions on the volatility function $F$ as in Garnier and Sølna [14]. In the $(\mathrm{LoH})$ case, we require that there exists some $\alpha>2$ such that

$$
\sum_{k=0}^{\infty} \frac{\alpha^{k} C_{k}^{2}}{k !}<\infty
$$

where the $C_{k}$ 's are the Hermite coefficients of the volatility function $F$ with respect to the invariant distribution of volatility factor $Z_{t}$ :

$$
\begin{aligned}
& C_{k}=\frac{1}{\sqrt{2 \pi}} \int_{\mathbb{R}} H_{k}(z) F^{2}\left(\sigma_{Z} z\right) \exp \left(-\frac{z^{2}}{2}\right) d z, \\
& H_{k}(z)=(-1)^{k} \exp \left(\frac{z^{2}}{2}\right) \frac{d^{k}}{d z^{k}} \exp \left(-\frac{z^{2}}{2}\right) .
\end{aligned}
$$

\subsection{A Special Model: The Fractional Ornstein-Uhlenbeck Process}

A typical model for the volatility factor is the fractional OrnsteinUhlenbeck (fOU) process. We describe here how this process can be represented in terms of a fractional Brownian motion. Because fractional Brownian motion can be expressed in terms of standard Brownian motion, we also arrive at an expression for the fOU process as a Volterra process that belongs to the classes described above.

A fractional Brownian motion ( $\mathrm{fBm}$ ) is a zero-mean Gaussian process $\left(W_{t}^{H}\right)_{t \in \mathbb{R}}$ with the covariance

$$
\mathbb{E}\left[W_{t}^{H} W_{s}^{H}\right]=\frac{\sigma_{H}^{2}}{2}\left(|t|^{2 H}+|s|^{2 H}-|t-s|^{2 H}\right),
$$

where $\sigma_{H}$ is a positive constant. We use the following movingaverage stochastic integral representation of the $\mathrm{fBm}$ (see [28])

$$
W_{t}^{H}=\frac{1}{\Gamma\left(H+\frac{1}{2}\right)} \int_{\mathbb{R}}\left((t-s)_{+}^{H-\frac{1}{2}}-(-s)_{+}^{H-\frac{1}{2}}\right) d W_{s},
$$

where $\left(W_{t}\right)_{t \in \mathbb{R}}$ is a standard Brownian motion over $\mathbb{R}$. Then $\left(W_{t}^{H}\right)_{t \in \mathbb{R}}$ is indeed a zero-mean Gaussian process with the covariance (15), and we have

$$
\begin{aligned}
\sigma_{H}^{2} & =\frac{1}{\Gamma\left(H+\frac{1}{2}\right)^{2}}\left[\int_{0}^{\infty}\left((1+s)^{H-\frac{1}{2}}-s^{H-\frac{1}{2}}\right)^{2} d s+\frac{1}{2 H}\right] \\
& =\frac{1}{\Gamma(2 H+1) \sin (\pi H)} .
\end{aligned}
$$

Let $\tau_{Z}>0$. We introduce the fOU process as

$$
\begin{aligned}
& Z_{t}=\tau_{Z}^{-H} \int_{-\infty}^{t} \exp \left(-\frac{t-s}{\tau_{Z}}\right) d W_{s}^{H} \\
& =\tau_{Z}^{-H} W_{t}^{H}-\tau_{Z}^{-1-H} \int_{-\infty}^{t} \exp \left(-\frac{t-s}{\tau_{Z}}\right) W_{s}^{H} d s .
\end{aligned}
$$

The fOU process is a fractional Brownian motion with a restoring force toward zero. It is a zero-mean, stationary Gaussian process, with variance

$$
\mathbb{E}\left[Z_{t}^{2}\right]=\sigma_{Z}^{2}, \text { with } \sigma_{Z}^{2}=\frac{1}{2} \Gamma(2 H+1) \sigma_{H}^{2}=\frac{1}{2 \sin (\pi H)},
$$

which is independent of $\tau_{Z}$, and covariance

$$
\mathbb{E}\left[Z_{t} Z_{t+s}\right]=\sigma_{Z}^{2} \mathcal{C}_{Z}\left(\frac{s}{\tau_{Z}}\right)
$$

which is a function of $s / \tau_{Z}$ only, with the normalized correlation function

$$
\begin{aligned}
\mathcal{C}_{Z}(s) & =\frac{1}{\Gamma(2 H+1)}\left[\frac{1}{2} \int_{\mathbb{R}}|s+v|^{2 H} \exp (-|v|) d v-|s|^{2 H}\right] \\
& =\frac{2 \sin (\pi H)}{\pi} \int_{0}^{\infty} \cos (s x) \frac{x^{1-2 H}}{1+x^{2}} d x .
\end{aligned}
$$

This shows that $\tau_{Z}$ is the mean-reversion time of the fOU process $Z_{t}$.

If $H=1 / 2$, then the process $Z_{t}$ is the standard OU process (synthesized with a standard Brownian motion). This process is a stationary Gaussian Markov process with an exponential correlation, and hence a mixing process:

$$
\mathcal{C}_{Z}(s)=\exp (-|s|)
$$

If $H \neq 1 / 2$, then the random process $Z_{t}$ is neither a martingale, nor a Markov process. For $H \in(0,1 / 2)$ it possesses short-range correlation properties in the sense that its correlation function is rough at zero:

$$
\mathcal{C}_{Z}(s)=1-\frac{1}{\Gamma(2 H+1)}|s|^{2 H}+o\left(|s|^{2 H}\right), \quad|s| \ll 1,
$$

while it is integrable and decays as $|s|^{2 H-2}$ at infinity.

For $H \in(1 / 2,1)$, it possesses long-range correlation properties

$$
\mathcal{C}_{Z}(s)=\frac{1}{\Gamma(2 H-1)}|s|^{2 H-2}+o\left(|s|^{2 H-2}\right), \quad|s| \gg 1,
$$

while it is of the form $\mathcal{C}_{Z}(s)=1-|s|^{2 H} / \Gamma(2 H+1)+o\left(|s|^{2 H}\right)$ at zero.

Using Equations (16) and (18), we arrive at the movingaverage integral representation of the fOU process as

$$
Z_{t}=\frac{\sigma_{Z}}{\sqrt{\tau_{Z}}} \int_{-\infty}^{t} \mathcal{K}\left(\frac{t-s}{\tau_{Z}}\right) d W_{s}
$$


where

$$
\mathcal{K}(t)=\frac{\sqrt{2 \sin (\pi H)}}{\Gamma\left(H+\frac{1}{2}\right)}\left[t^{H-\frac{1}{2}}-\int_{0}^{t}(t-s)^{H-\frac{1}{2}} \exp (-s) d s\right] .
$$

The main properties of the kernel $\mathcal{K}$ in our context are the following ones:

$-\mathcal{K} \in L^{2}(0, \infty)$ with $\int_{0}^{\infty} \mathcal{K}^{2}(u) d u=1$.

$-\mathcal{K} \in L^{1}(0, \infty)$ if $H \in(0,1 / 2]$

$-\mathcal{K} \in L^{\infty}(0, \infty)$ if $H \in[1 / 2,1)$,

- for $t \ll 1$,

$$
\mathcal{K}(t)=\frac{\sqrt{2 \sin (\pi H)}}{\Gamma\left(H+\frac{1}{2}\right)}\left(t^{H-\frac{1}{2}}+O\left(t^{H+\frac{1}{2}}\right)\right),
$$

- for $H \neq 1 / 2$ and for $t \gg 1$,

$$
\mathcal{K}(t)=\frac{\sqrt{2 \sin (\pi H)}}{\Gamma\left(H-\frac{1}{2}\right)}\left(t^{H-\frac{3}{2}}+O\left(t^{H-\frac{5}{2}}\right)\right) .
$$

This shows that the fOU process belongs to the class $(\mathrm{RoH})$ if $H<1 / 2$ with $d_{Z}=\sqrt{2 \sin (\pi H)} / \Gamma(H+1 / 2)$, to the class (St) if $H=1 / 2$, and to the class $(\mathrm{LoH})$ if $H>1 / 2$ with $c_{Z}=\sqrt{2 \sin (\pi H)} / \Gamma(H-1 / 2)$.

We remark that the fOU process inherits the regularity properties of the fractional Brownian motion process so that it is Hölder continuous with exponent $\gamma$ for any $\gamma<H$.

\subsection{Covariance Properties of the Stochastic Volatility}

We return to the general volatility factor model of section 3.1 and discuss the correlation properties of the associated volatility $\sigma_{t}=$ $F\left(Z_{t}\right)$. As discussed above, the volatility factor $Z_{t}$ possesses shortrange or long-range correlation properties. As we now show, the volatility process $\sigma_{t}$ inherits these properties.

Lemma 3.1. We denote, for $j=1,2$,

$$
\left\langle F^{j}\right\rangle=\int_{\mathbb{R}} F\left(\sigma_{Z} z\right)^{j} p(z) d z, \quad\left\langle F^{\prime j}\right\rangle=\int_{\mathbb{R}} F^{\prime}\left(\sigma_{Z} z\right)^{j} p(z) d z,
$$

where $p(z)$ is the $p d f$ of the standard normal distribution.

1. The process $\sigma_{t}$ is a stationary random process with mean $\mathbb{E}\left[\sigma_{t}\right]=\langle F\rangle$ and variance $\operatorname{Var}\left(\sigma_{t}\right)=\left\langle F^{2}\right\rangle-\langle F\rangle^{2}$.

2. The covariance function of the process $\sigma_{t}$ is of the form

$$
\operatorname{Cov}\left(\sigma_{t}, \sigma_{t+s}\right)=\left(\left\langle F^{2}\right\rangle-\langle F\rangle^{2}\right) \mathcal{C}_{\sigma}(s),
$$

where the correlation function $\mathcal{C}_{\sigma}$ satisfies $\mathcal{C}_{\sigma}(0)=1$.

If the volatility factor belongs to the class $(\mathrm{RoH})$ with $\mathrm{H}<$ $1 / 2$, then $\mathcal{C}_{\sigma}$ is integrable and we have

$$
\mathcal{C}_{\sigma}(s)=1-\frac{q_{Z}\left\langle F^{\prime 2}\right\rangle}{\left\langle F^{2}\right\rangle-\langle F\rangle^{2}}|s|^{2 H}+o\left(|s|^{2 H}\right) \quad \text { for }|s| \ll 1,
$$

which shows that $\sigma_{t}$ has short-range correlation properties.
If the volatility factor belongs to the class $(\mathrm{LoH})$ with $\mathrm{H}>$ $1 / 2$, then $\mathcal{C}_{\sigma}$ is not integrable,

$$
\mathcal{C}_{\sigma}(s)=\frac{k_{Z}\left\langle F^{\prime}\right\rangle^{2}}{\left\langle F^{2}\right\rangle-\langle F\rangle^{2}}|s|^{2 H-2}+o\left(|s|^{2 H-2}\right) \quad \text { for }|s| \gg 1,(30)
$$

which shows that $\sigma_{t}$ has long-range correlation properties. Moreover, $\mathcal{C}_{\sigma}$ does not have short-range correlation properties.

If the volatility factor belongs to the class $(S t)$, then $\mathcal{C}_{\sigma}$ does not have short-range or long-range correlation properties. Its behavior at zero is $\mathcal{C}_{\sigma}(s)=1-O(|s|)$ and it is integrable. The proof of the lemma can be found in Garnier and Sølna [12, 13].

\section{SCALES}

Our goal is to compute the option price defined as the martingale

$$
M_{t}=\mathbb{E}\left[h\left(X_{T}\right) \mid \mathcal{F}_{t}\right],
$$

where $t \leq T$ and $h$ is a smooth payoff function with bounded derivatives apart from a finite set of points where it may have jump discontinuities in its derivatives. In the general case of the volatility factor introduced in section 3.1 there is no explicit expression for this price. However, we will show that we can identify asymptotic approximations of the price if we exploit separation in the characteristic time scales associated with the model. We can identify three time scales:

- the time to maturity

$$
\tau=T-t
$$

- the mean-reversion time of the stochastic volatility $\tau_{Z}$,

- the diffusion time

$$
\tau_{\sigma}=\frac{2}{\bar{\sigma}^{2}},
$$

with $\bar{\sigma}^{2}=\mathbb{E}\left[\sigma_{t}^{2}\right]=\mathbb{E}\left[F\left(Z_{t}\right)^{2}\right]$ or

$$
\bar{\sigma}^{2}=\left\langle F^{2}\right\rangle=\int_{\mathbb{R}} F\left(\sigma_{Z} z\right)^{2} p(z) d z .
$$

We discuss next asymptotic characterizations for the option price depending on the relative magnitudes of these time scales. These results follow via modifications of arguments presented in Garnier and Sølna [12-14]. The asymptotic analysis of the option prices presented there involves the martingale method which exploits the fact that asset price process is a martingale and seeks to construct a decomposition that is a martingale plus a small correction. We shall consider the case when $\tau_{\sigma}$ and $\tau$ are of the same order, corresponding to the relative asset fluctuations being of order one at maturity. The important scaling parameter in our formulation is $\tau_{Z} / \tau_{\sigma}$ and we consider the two cases when this is small, respectively large, corresponding to fast, respectively slow, mean reversion for the volatility process. 


\section{OPTION PRICE}

We introduce the operator

$$
\mathcal{L}_{\mathrm{BS}}(\sigma)=\partial_{s}+\frac{1}{2} \sigma^{2} x^{2} \partial_{x}^{2}
$$

that is, the standard Black-Scholes operator with zero interest rate and constant volatility $\sigma$. We also introduce the option price $Q_{s}^{(0)}(x ; \sigma)$ given by the Black-Scholes formula in the case of zero interest rate and constant volatility $\sigma$ (see [1]):

$$
\mathcal{L}_{\mathrm{BS}}(\sigma) Q_{s}^{(0)}(x ; \sigma)=0, \quad s \in(0, T), \quad Q_{T}^{(0)}(x ; \sigma)=h(x) .
$$

\subsection{Slow Regime}

We address the regime where

$$
\delta=\frac{\tau_{\sigma}}{\tau_{Z}}
$$

is small and the time to maturity $\tau$ is of the same order as $\tau_{\sigma}$. This corresponds to a regime where the volatility factor evolves on a time scale that is short relative to the time to maturity. The following proposition revisits Proposition 6.1 in Garnier and Sølna [12] in the case of a general Volterra process.

Proposition 5.1. When $\delta$ is small, if the volatility factor belongs to one of the three classes and if the volatility factor satisfies (7) for some $H \in(0,1)$, then the option price (31) is of the form

$$
M_{t}=Q_{t}\left(X_{t}\right)+o\left(\delta^{H}\right)
$$

where

$$
Q_{t}(x)=Q_{t}^{(0)}\left(x ; \sigma_{t}\right)+p_{t} q_{t} \phi_{t, T}\left[x^{2} \partial_{x}^{2} Q_{t}^{(0)}\left(x ; \sigma_{t}\right)\right]+\rho p_{t}^{2} q_{t} Q_{t}^{(1)}(x),
$$

$p_{t}=F\left(Z_{t}\right) / \bar{\sigma}, q_{t}=F^{\prime}\left(Z_{t}\right) / \bar{\sigma}, Q_{t}^{(0)}\left(x ; \sigma_{t}\right)$ is the Black-Scholes formula (36) with constant volatility $\sigma_{t}=\bar{\sigma} p_{t}, \phi_{t, T}$ is the random component

$$
\phi_{t, T}=\bar{\sigma}^{2} \mathbb{E}\left[\int_{t}^{T} Z_{s}-Z_{t} d s \mid \mathcal{F}_{t}\right]
$$

and $Q_{t}^{(1)}(x)$ is the correction

$$
Q_{t}^{(1)}(x)=\left(x \partial_{x}\left(x^{2} \partial_{x}^{2} Q_{t}^{(0)}\left(x ; \sigma_{t}\right)\right)\right) D_{T-t},
$$

with $D_{T-t}$ defined by

$$
D_{T-t}=\bar{D}(T-t)^{H+\frac{3}{2}}, \quad \bar{D}=\frac{\bar{\sigma}^{3} \sigma_{Z} d_{Z}}{\left(H+\frac{1}{2}\right)\left(H+\frac{3}{2}\right) \tau_{Z}^{H}} .
$$

The expansion (52) is in the sense that:

$$
\limsup _{\delta \rightarrow 0} \delta^{-H} \sup _{t \in[0, T]} \mathbb{E}\left[\left|M_{t}-Q_{t}\left(X_{t}\right)\right|^{2}\right]^{\frac{1}{2}}=0 .
$$

The hypothesis "the volatility factor satisfies (7) for some $H \in$ $(0,1)$ " means that there exist $H \in(0,1)$ and $d_{Z} \neq 0$ so that $\mathcal{K}(t)=d_{Z} t^{H-1 / 2}(1+o(1))$ as $t \rightarrow 0$. For instance, the standard $(H=1 / 2)$ and fractional $(H \neq 1 / 2)$ OU processes satisfy this hypothesis by (25).

The parameter $D_{T-t}$ is of order $\tau_{\sigma}^{-3 / 2} \tau^{H+3 / 2} \tau_{Z}^{-H} \sim \delta^{H}$ (note that $\bar{\sigma}^{3} \sim \tau_{\sigma}^{-3 / 2}$ ). The random variable $\phi_{t, T}$ is Gaussian with mean zero and variance

$$
\mathbb{E}\left[\phi_{t, T}^{2}\right]=\frac{\bar{\sigma}^{4} \sigma_{Z}^{2} d_{Z}^{2} \alpha_{H}(T-t)^{2+2 H}}{\tau_{Z}^{2 H}\left(H+\frac{1}{2}\right)^{2}}+o\left(\delta^{2 H}\right),
$$

with

$$
\alpha_{H}=\int_{0}^{\infty}\left[(1+v)^{H+\frac{1}{2}}-v^{H+\frac{1}{2}}-\left(H+\frac{1}{2}\right) v^{H-\frac{1}{2}}\right]^{2} d v,
$$

which is positive for any $H \in(0,1 / 2) \cup(1 / 2,1)$ and is zero for $H=1 / 2$. This shows that $\mathbb{E}\left[\phi_{t, T}^{2}\right]$ is of order $\tau_{\sigma}^{-2} \tau_{Z}^{-2 H} \tau^{2+2 H} \sim$ $\delta^{2 H}$ and $\phi_{t, T}$ is of order $\delta^{H}$. Thus, the two corrective terms in (39) are of order $\delta^{H}$. Finally, we remark that it is possible to replace $p_{t}$ and $q_{t}$ by $p_{0}$ and $q_{0}$ in (39) without changing the order of magnitude of the remainder in (38). However, it is important to compute the Black-Scholes price at time $t$ with the volatility $\sigma_{t}$ at time $t$ as stated in the proposition.

Proof: By following [12], we find that the option price (31) is of the form

$$
M_{t}=\tilde{Q}_{t}\left(X_{t}\right)+o\left(\delta^{H}\right)
$$

where

$$
\tilde{Q}_{t}(x)=\tilde{Q}_{t}^{(0)}(x)+p_{0} q_{0} \tilde{\phi}_{t, T}\left[x^{2} \partial_{x}^{2} \tilde{Q}_{t}^{(0)}(x)\right]+\rho p_{0}^{2} q_{0} \tilde{Q}_{t}^{(1)}(x),
$$

$p_{0}=F\left(Z_{0}\right) / \bar{\sigma}, q_{0}=F^{\prime}\left(Z_{0}\right) / \bar{\sigma}, \tilde{Q}_{t}^{(0)}(x)$ is given by the BlackScholes formula with constant volatility $\sigma_{0}=\bar{\sigma} p_{0}: \tilde{Q}_{t}^{(0)}(x)=$ $Q_{t}^{(0)}\left(x ; \sigma_{0}\right), \tilde{\phi}_{t, T}$ is the random component

$$
\tilde{\phi}_{t, T}=\bar{\sigma}^{2} \mathbb{E}\left[\int_{t}^{T} Z_{s}-Z_{0} d s \mid \mathcal{F}_{t}\right]
$$

and $\tilde{Q}_{t}^{(1)}(x)$ is the correction

$$
\tilde{Q}_{t}^{(1)}(x)=\left(x \partial_{x}\left(x^{2} \partial_{x}^{2} \tilde{Q}_{t}^{(0)}(x)\right)\right) D_{T-t},
$$

with $D_{T-t}$ defined by (42). The random variable $\tilde{\phi}_{t, T}$ is Gaussian with mean zero and variance

$$
\begin{aligned}
\mathbb{E}\left[\tilde{\phi}_{t, T}^{2}\right]= & \frac{\bar{\sigma}^{4} \sigma_{Z}^{2} d_{Z}^{2} T^{2+2 H}}{\tau_{Z}^{2 H}\left(H+\frac{1}{2}\right)^{2}} \int_{0}^{\infty}\left[\left(1-\frac{t}{T}+v\right)^{H+\frac{1}{2}}-v^{H+\frac{1}{2}}\right. \\
& \left.-\left(1-\frac{t}{T}\right)\left(H+\frac{1}{2}\right)\left(v-\frac{t}{T}\right)_{+}^{H-\frac{1}{2}}\right]^{2} d v+o\left(\delta^{2 H}\right) .
\end{aligned}
$$


In fact, it is possible to improve this result in order to get the proposition. For any $\sigma$, the $\sigma$-derivative of the solution of $\mathcal{L}_{\mathrm{BS}}(\sigma) Q_{s}^{(0)}(x ; \sigma)=0$ with the terminal condition $Q_{T}^{(0)}(x ; \sigma)=$ $h(x)$ satisfies

$$
\begin{aligned}
& \mathcal{L}_{\mathrm{BS}}(\sigma)\left[\partial_{\sigma} Q_{s}^{(0)}(x ; \sigma)\right]=-\sigma x^{2} \partial_{x}^{2} Q_{s}^{(0)}(x ; \sigma), \quad s \in(0, T), \\
& \partial_{\sigma} Q_{T}^{(0)}(x ; \sigma)=0 .
\end{aligned}
$$

Thus, it is of the form:

$$
\partial_{\sigma} Q_{t}^{(0)}(x ; \sigma)=\sigma(T-t) x^{2} \partial_{x}^{2} Q_{t}^{(0)}(x ; \sigma) .
$$

We then find

$$
\begin{aligned}
\tilde{Q}_{t}^{(0)}(x) & =Q_{t}^{(0)}\left(x ; \sigma_{0}\right)=Q_{t}^{(0)}\left(x ; \sigma_{t}\right)+\left(\sigma_{0}-\sigma_{t}\right) \partial_{\sigma} Q_{t}^{(0)}\left(x ; \sigma_{t}\right) \\
& +o\left(\delta^{H}\right) \\
& =Q_{t}^{(0)}\left(x ; \sigma_{t}\right)+\bar{\sigma}^{2} p_{t} q_{t}(T-t) x^{2} \partial_{x}^{2} Q_{t}^{(0)}\left(x ; \sigma_{t}\right)\left(Z_{0}-Z_{t}\right) \\
& +o\left(\delta^{H}\right), \\
\tilde{Q}_{t}^{(1)}(x) & =Q_{t}^{(1)}(x)+o\left(\delta^{H}\right),
\end{aligned}
$$

which gives the desired result.

As mentioned above, the two corrective terms in (39) are of order $\delta^{H}$. The first one (proportional to $\phi_{t, T}$ ) has mean zero and Gaussian statistics, the second one (proportional to $Q_{t}^{(1)}$ ) is deterministic.

If the volatility factor is a fOU process with Hurst index $H$, then $\bar{D}$ is given by

$$
\bar{D}=\frac{\bar{\sigma}^{3}}{\Gamma\left(H+\frac{5}{2}\right) \tau_{Z}^{H}},
$$

and the variance of $\phi_{t, T}$ is

$$
\mathbb{E}\left[\phi_{t, T}^{2}\right]=\frac{\bar{\sigma}^{4} \alpha_{H}(T-t)^{2+2 H}}{\tau_{Z}^{2 H} \Gamma\left(H+\frac{3}{2}\right)^{2}}+o\left(\delta^{2 H}\right) .
$$

For the standard OU process $\left(H=1 / 2, \sigma_{Z}=1 / \sqrt{2}, d_{Z}=\right.$ $\sqrt{2})$, we have $D_{T-t}=\frac{\bar{\sigma}^{3}(T-t)^{2}}{2 \tau_{Z}^{1 / 2}}+o(\sqrt{\delta})$ and $\mathbb{E}\left[\phi_{t, T}^{2}\right]=0+o(\delta)$ (because $\alpha_{H}=0$ ). In the standard case [class $(S t)$ ] the term $\phi_{t, T}$ is negligible.

Thus, we have a universal characterization of the price in this regime of a slow volatility factor. We remark that the price correction is larger for a rougher volatility factor corresponding a smaller Hurst index $H$.

\subsection{Fast Regime}

We consider the regime where

$$
\varepsilon=\frac{\tau_{Z}}{\tau_{\sigma}}
$$

is small and the time to maturity $\tau$ is of the same order as $\tau_{\sigma}$. This corresponds to a regime where the volatility factor evolves on a time scale that is fast relative to the time to maturity. We first address the case where the volatility factor is standard or rough. The following proposition is a reformulation of Proposition 1 in Garnier and Sølna [13].
Proposition 5.2. If the volatility factor belongs to the class $(\mathrm{RoH})$ with $H<1 / 2$ or to the class (St), then we have

$$
M_{t}=Q_{t}\left(X_{t}\right)+o\left(\varepsilon^{\frac{1}{2}}\right)
$$

where

$$
Q_{t}(x)=Q_{t}^{(0)}(x ; \bar{\sigma})+\rho Q_{t}^{(1)}(x)
$$

$Q_{t}^{(0)}(x ; \bar{\sigma})$ is deterministic and given by the Black-Scholes formula (36) with constant volatility $\bar{\sigma}, Q_{t}^{(1)}(x)$ is the deterministic correction

$$
Q_{t}^{(1)}(x)=\left(x \partial_{x}\left(x^{2} \partial_{x}^{2} Q_{t}^{(0)}(x ; \bar{\sigma})\right)\right) D_{T-t}, \quad D_{T-t}=(T-t) \bar{D},
$$

$\bar{D}$ is the coefficient defined by

$$
\bar{D}=\sigma_{Z} \tau_{Z}^{\frac{1}{2}} \int_{0}^{\infty}\left[\iint_{\mathbb{R}^{2}} F\left(\sigma_{Z} z\right)\left(F F^{\prime}\right)\left(\sigma_{Z} z^{\prime}\right) p_{\mathcal{C}_{Z}(s)}\left(z, z^{\prime}\right) d z d z^{\prime}\right] \mathcal{K}(s) d s,
$$

$p_{C}\left(z, z^{\prime}\right)$ is the pdf of the bivariate normal distribution with mean zero and covariance matrix $\left(\begin{array}{ll}1 & C \\ C & 1\end{array}\right)$.

The expansion (52) is in the sense:

$$
\lim _{\varepsilon \rightarrow 0} \varepsilon^{-\frac{1}{2}} \sup _{t \in[0, T]} \mathbb{E}\left[\left|M_{t}-Q_{t}\left(X_{t}\right)\right|^{2}\right]^{\frac{1}{2}}=0 .
$$

The coefficient $D_{T-t}$ is of order $\tau_{\sigma}^{-3 / 2} \tau_{Z}^{1 / 2} \tau \sim \varepsilon^{1 / 2}$ (note that $F$ and $F^{\prime}$ are propositional to $\bar{\sigma} \sim \tau_{\sigma}^{-1 / 2}$ ). Thus, the corrective term in (53) is of order $\varepsilon^{1 / 2}$ and deterministic.

If the function $F$ is exponential:

$$
F(z)=\bar{\sigma} \exp \left(z-\sigma_{Z}^{2}\right)
$$

then we can get a simplified expression for $\bar{D}$ (although the function violates the technical assumption that $F$ should be bounded):

$$
\bar{D}=\bar{\sigma}^{3} \sigma_{Z} \tau_{Z}^{\frac{1}{2}} \exp \left(-\frac{\sigma_{Z}^{2}}{2}\right) \int_{0}^{\infty} \exp \left(-2 \sigma_{Z}^{2} \mathcal{C}_{Z}(s)\right) \mathcal{K}(s) d s
$$

which gives $\bar{D}=\bar{\sigma}^{3} \tau_{Z}^{1 / 2}[\exp (3 / 4)-\exp (-1 / 4)]$ if, additionally, the volatility factor is a standard OU process.

This proposition shows that the asymptotic expansion of the option price has the same form as in the mixing case addressed in Fouque et al. [1,46]. When the stochastic volatility is fast-varying, its short-range correlation property is not visible to leading order nor in the first correction. In other words, in the rapid mean reversion regime, the roughness of the process does not affect the qualitative form of the price correction. This is in contrast to the slowly-varying case addressed in the previous section, and in contrast to the situation where the stochastic volatility has longrange correlation properties as seen in the following proposition (which is an extension of Proposition 4.1 in [14]). 
Proposition 5.3. If the volatility factor belongs to the class $(\mathrm{LoH})$ with $H>1 / 2$, then we have

$$
M_{t}=Q_{t}\left(X_{t}\right)+o\left(\varepsilon^{1-H}\right)
$$

where

$$
Q_{t}(x)=Q_{t}^{(0)}(x ; \bar{\sigma})+\left(x^{2} \partial_{x}^{2} Q_{t}^{(0)}(x ; \bar{\sigma})\right) \phi_{t, T}+\rho Q_{t}^{(1)}(x) .
$$

The function $Q_{t}^{(0)}(x ; \bar{\sigma})$ is deterministic and given by the BlackScholes formula (36) with constant volatility $\bar{\sigma}$. The random component $\phi_{t, T}$ is given by

$$
\phi_{t, T}=\mathbb{E}\left[\frac{1}{2} \int_{t}^{T}\left(\sigma_{s}^{2}-\bar{\sigma}^{2}\right) d s \mid \mathcal{F}_{t}\right]
$$

The function $Q_{t}^{(1)}(x)$ is the deterministic correction

$$
Q_{t}^{(1)}(x)=\left(x \partial_{x}\left(x^{2} \partial_{x}^{2} Q_{t}^{(0)}(x ; \bar{\sigma})\right)\right) D_{T-t}
$$

with $D_{T-t}$ defined by

$$
D_{T-t}=\bar{D}(T-t)^{H+\frac{1}{2}}, \quad \bar{D}=\frac{\langle F\rangle\left\langle F F^{\prime}\right\rangle \sigma_{Z} c_{Z} \tau_{Z}^{1-H}}{\left(H+\frac{1}{2}\right)\left(H-\frac{1}{2}\right)} .
$$

The coefficient $D_{T-t}$ is of order $\tau_{\sigma}^{-3 / 2} \tau_{Z}^{1-H} \tau^{H+1 / 2} \sim \varepsilon^{1-H}$ (note that $\langle F\rangle\left\langle F F^{\prime}\right\rangle$ is of order $\bar{\sigma}^{3} \sim \tau_{\sigma}^{-3 / 2}$ ).

The random variable $\phi_{t, T}$ has mean zero and variance

$$
\begin{aligned}
& \mathbb{E}\left[\phi_{t, T}^{2}\right]=\frac{\left\langle F F^{\prime}\right\rangle^{2} \sigma_{Z}^{2} c_{Z}^{2} \tau_{Z}^{2-2 H}(T-t)^{2 H}}{\left(H-\frac{1}{2}\right)^{2}} \\
& \times\left(\frac{\Gamma\left(H+\frac{1}{2}\right)^{2}}{\Gamma(2 H+1) \sin (\pi H)}-\frac{1}{2 H}\right)+o\left(\varepsilon^{2-2 H}\right),
\end{aligned}
$$

which is of order $\tau_{\sigma}^{-2} \tau_{Z}^{2-2 H} \tau^{2 H} \sim \varepsilon^{2-2 H}$ (note that $\left\langle F F^{\prime}\right\rangle^{2}$ is of order $\bar{\sigma}^{4} \sim \tau_{\sigma}^{-2}$ ). Moreover, after normalization by its standard deviation, it converges in distribution to a standard Gaussian random variable when $\varepsilon$ goes to 0 . Further results on the correlation structure of $\phi_{t, T}$ as a non-stationary random field in $(t, T)$ are given in Garnier and Sølna [14].

The two corrective terms in (57) (proportional to $\phi_{t, T}$ and $\left.Q_{t}^{(1)}\right)$ are of the same order $\varepsilon^{1-H}$. The first one is random, zeromean and approximately Gaussian distributed, and the second one is deterministic.

If the volatility factor is a fOU process with Hurst index $H$, then

$$
\bar{D}=\frac{\langle F\rangle\left\langle F F^{\prime}\right\rangle \tau_{Z}^{1-H}}{\Gamma\left(H+\frac{3}{2}\right)},
$$

and.

$$
\begin{aligned}
& \mathbb{E}\left[\phi_{t, T}^{2}\right]=\left\langle F F^{\prime}\right\rangle^{2} \tau_{Z}^{2-2 H}(T-t)^{2 H} \\
& \times\left(\frac{1}{\Gamma(2 H+1) \sin (\pi H)}-\frac{1}{2 H \Gamma\left(H+\frac{1}{2}\right)^{2}}\right)+o\left(\varepsilon^{2-2 H}\right) .
\end{aligned}
$$

\section{IMPLIED VOLATILITY}

For a European call option $h(x)=(x-K)_{+}$we have from Equation (1.41) in [1]:

$$
\begin{aligned}
& Q_{t}^{(0)}(x ; \sigma)=x \Phi\left(\frac{1}{\sigma \sqrt{T-t}} \log \left(\frac{x}{K}\right)+\frac{\sigma \sqrt{T-t}}{2}\right) \\
& -K \Phi\left(\frac{1}{\sigma \sqrt{T-t}} \log \left(\frac{x}{K}\right)-\frac{\sigma \sqrt{T-t}}{2}\right),
\end{aligned}
$$

where $\Phi$ is the cumulative distribution function of the standard normal distribution.

In this section, we define the implied volatility as the volatility that, when used in the constant volatility Black-Scholes pricing formula, gives the same price as the price approximation in the considered regime, to the order of the approximation. We give in the following the expressions for the implied volatility in the different regimes addressed in the previous section. As we will see the implied volatility as a function of time to maturity has fractional behaviors.

\subsection{Slow Regime}

Proposition 6.1. Under the conditions of Proposition 5.1, the implied volatility in the context of the European call option is given by

$$
\begin{aligned}
I_{t} & =\sigma_{t}+\bar{\sigma}\left\{q_{t} \frac{\phi_{t, T}}{\bar{\sigma}^{2}(T-t)}+\frac{\rho q_{t} \sigma_{Z} d_{Z}}{\left(H+\frac{1}{2}\right)\left(H+\frac{3}{2}\right) \tau_{Z}^{H}}\left[\frac{p_{t}}{2}(T-t)^{H+\frac{1}{2}}\right.\right. \\
& +\frac{\log \left(K / X_{t}\right)}{\left.\left.\bar{\sigma}^{2} p_{t}(T-t)^{\frac{1}{2}-H}\right]+o\left(\delta^{H}\right)\right\},}
\end{aligned}
$$

where $\phi_{t, T}$ is defined by (40) (that does not depend on $X_{t}$ ), $p_{t}=$ $F\left(Z_{t}\right) / \bar{\sigma}$ and $q_{t}=F^{\prime}\left(Z_{t}\right) / \bar{\sigma}$.

The two terms proportional to $q_{t}$ in Equation (65) are of order $\delta^{H}$. In the standard case (class $(S t)$ ), the term $\phi_{t, T}$ is negligible. In the general case, the first two terms can be combined and rewritten as:

$$
\sigma_{t}+\bar{\sigma}\left\{q_{t} \frac{\phi_{t, T}}{\bar{\sigma}^{2}(T-t)}\right\}=\mathbb{E}\left[\frac{1}{T-t} \int_{t}^{T} \sigma_{s}^{2} d s \mid \mathcal{F}_{t}\right]^{\frac{1}{2}}+o\left(\delta^{H} \bar{\sigma}\right) .
$$

We can see from Equation (65) that, if $H \in(0,1 / 2)$, then the skew of the implied volatility, $\left(\partial I_{t} / \partial X_{t}\right)$, diverges as $(T-t)^{H-1 / 2}$ for short time to maturity, as was observed by Alòs et al. [39] for a fOU process. It also follows that if $H \in(1 / 2,1)$, then the skew goes to zero, as was also observed by Alòs et al. [39] for a fOU process.

\subsection{Fast Regime}

Proposition 6.2. Under the conditions of Proposition 5.2, the implied volatility in the context of the European option when the volatility factor belongs to the class $(\mathrm{St})$ or $(\mathrm{RoH})$ is given by

$$
I_{t}=\bar{\sigma}\left\{1+\frac{\rho \bar{D}}{\bar{\sigma}^{2}}\left[\frac{1}{2}+\frac{\log \left(\frac{K}{X_{t}}\right)}{\bar{\sigma}^{2}(T-t)}\right]+o\left(\varepsilon^{\frac{1}{2}}\right)\right\},
$$

where $\bar{D}$ is defined by (55). 
The corrective term (proportional to $\bar{D}$ ) is of order $\varepsilon^{1 / 2}$. The expression (67) obtained for the class $(\mathrm{RoH})$ of rough stochastic volatility models has the same form as the expression obtained in [[46], Equation (5.55)] with a stochastic volatility that is an ordinary OU process, that is, a Markov process with exponentially decaying correlations. This proposition shows that a rough volatility does not change the form of the implied volatility in the fast mean reversion regime compared to the standard Markov case.

Proposition 6.3. Under the conditions of Proposition 5.3, the implied volatility in the context of the European option when the volatility factor belongs to the class $(\mathrm{LoH})$ with $H \in(1 / 2,1)$ is given by

$$
\begin{aligned}
& I_{t}=\bar{\sigma}\left\{1+\frac{\phi_{t, T}}{\bar{\sigma}^{2}(T-t)}+\frac{\rho \bar{D}}{\bar{\sigma}^{2}}\left[\frac{1}{2}(T-t)^{H-\frac{1}{2}}\right.\right. \\
& \left.\left.+\log \left(\frac{K}{X_{t}}\right) \frac{(T-t)^{H-\frac{3}{2}}}{\bar{\sigma}^{2}}\right]+o\left(\varepsilon^{1-H}\right)\right\},
\end{aligned}
$$

where $\phi_{t, T}$ is defined by (58) (that does not depend on $X_{t}$ ) and $\bar{D}$ is defined by (60).

The two corrective terms (the one proportional to $\phi_{t, T}$ and the one proportional to $\bar{D}$ ) are of order $\varepsilon^{1-H}$. The first two terms can be combined and rewritten as:

$$
\bar{\sigma}\left\{1+\frac{\phi_{t, T}}{\bar{\sigma}^{2}(T-t)}\right\}=\mathbb{E}\left[\frac{1}{T-t} \int_{t}^{T} \sigma_{s}^{2} d s \mid \mathcal{F}_{t}\right]^{\frac{1}{2}}+o\left(\varepsilon^{1-H} \bar{\sigma}\right) .
$$

Note that the skew of the implied volatility goes to zero for long time to maturity, but the value of the implied volatility (atthe-money or out-of-the-money) diverges as $(T-t)^{H-1 / 2}$. This behavior is in dramatic contrast to the one in the standard and rough cases described in Proposition 6.2.

\section{REMARKS ON CALIBRATION}

In the fast regime case, with $H \leq 1 / 2$, the parameters $\rho \bar{D}, \bar{\sigma}$ can be calibrated from the observations of a few liquid option prices for different strikes and time to maturities via (67). We refer to Fouque et al. [1] for an illustration of such calibration.

In the fast regime case, with $H>1 / 2$, the parameters $\rho \bar{D}, \bar{\sigma}$ and $H$ can be calibrated from the observations of a set of liquid options by (68) and by using the fact that $\phi_{t, T}$ has mean zero. Note also that when $H>1 / 2$, the correlation function of the Gaussian random component $\phi_{t, T} / \bar{\sigma}$ is known and depends only on $H$ (see [14]), so that it is possible to estimate the implied volatility surface from the observations by Gaussian conditioning. We remark that the random component $\phi_{t, T} / \bar{\sigma}$ explains the random fluctuations that are sometimes seen for the at-the-money implied volatility as function of time to maturity. Moreover, the fractional dependence on time to maturity is characteristic of implied volatility data (see [1]).

In the slow regime case the form of the implied volatility is given by (65) for the three classes $(\mathrm{RoH}),(\mathrm{St})$ and $(\mathrm{LoH})$. The calibration procedure then may not seem so simple because $\sigma_{t}$, $p_{t}, q_{t}$, and $\phi_{t, T}$ depend on $\mathcal{F}_{t}$. However, this reflects the common context that models are typically recalibrated as new data become available and that market parameters exhibit modulation on a short time scale relative to say a tick-by-tick scale. What we have done here is to present a consistent multiscale framework where the slow modulation parameters reflect the local stationarity of the market and of the implied surface and how to still carry out a consistent calibration. To this effect note that to the order of the approximation we can replace $p_{t}, q_{t}$ by $p_{0}, q_{0}$ in (65) giving a fixed skew in $\log$-moneyness $\log (K / X)$. It is however important to capture the at-the-money fluctuations of the implied volatility as articulated by $\sigma_{t}$ and $\phi_{t, T}$. Thus, we have provided a framework where the recalibration can be carried out by differentiating the local modulation parameters $\sigma_{t}$ and $\phi_{t, T}$ and the global structural parameters that determine the implied volatility surface.

It is noteworthy that the maturity modulation in the logmoneyness term $\log (K / X)$ is qualitatively different in the slow and fast cases and in fact can be used to distinguish these regimes. We remark that modeling with a two-factor stochastic volatility model (with one slow factor and one fast factor) may be appropriate.

\section{CONCLUSIONS}

We have considered a class of fractional stochastic volatility models. The stochastic volatility process is modeled as a smooth deterministic function of a stationary fractional Gaussian process, a Volterra process. The Volterra process can be expressed as a moving average of a standard Brownian motion. Depending on the properties of the integration kernel in the moving average representation we identify and study three cases. First, the rough $(\mathrm{RoH})$ case corresponding to rough paths for the Volterrra process. In this case the autocorrelation function of the Volterra process decays faster than linearly at zero. Second, the standard or classic (St) case with autocorrelation function that decays linearly at zero and is integrable. Third, the long-range $(\mathrm{LoH})$ case characterized by slowly decaying correlations at infinity so that the associated autocorrelation function is not integrable. The two main asymptotic regimes we consider is when the mean reversion time of the stochastic volatility is short, respectively long, relative to the diffusion time scale. Here, the mean reversion time corresponds essentially to the time scale at which the volatility process decorrelates, while the characteristic diffusion time is essentially the inverse of the mean square volatility. The asymptotic analysis leads to simple parametric models for the implied volatility. The main qualitative insight is that in the rough case the behavior of the implied volatility is as in the classic Markov case corresponding to the (St) class with rapid mean reversion. However, both the rough $(\mathrm{RoH})$ case with slow mean reversion and the long-range $(\mathrm{LoH})$ case with slow or rapid mean reversion are qualitatively different. Then the power law tail in the volatility correlations leads to a price approximation that depends on the time to maturity to a fractional power determined by the power law of the autocorrelation function. We show here that in the short-range case the rough behavior gives rise to an implied volatility that diverges as the time to maturity goes to zero (as well as its 
skew), moreover, that in the long-range case the slow decay in the correlations gives a term structure of the implied volatility that diverges as the time to maturity goes to infinity (but its skew goes to zero) (see also $[13,14]$ ).

From the point of view of calibration the fact that the fractional stochastic volatility produces, in certain regimes, a fractional term structure (fractional behavior in time to maturity) for the implied volatility is important. This is indeed a behavior that is seen in market-data [47] so that having a modeling framework that produces such behavior is desirable.

We finally remark that here we have only considered application to option pricing. There are other applications of

\section{REFERENCES}

1. Fouque J-P, Papanicolaou G, Sircar KR, Sølna K. Multiscale Stochastic Volatility for Equity, Interest Rate, and Credit Derivatives. Cambridge: Cambridge University Press (2011).

2. Gatheral J. The Volatility Surface: a Practitioner's Guide. Hoboken, NJ: Wiley (2006).

3. Ghysels E, Harvey A, Renaud E. Stochastic volatility. In: Maddala GS, Rao CR, Editors. Handbook of Statistics. Vol. 14, Statistical Methods in Finance. Amsterdam (1995). p. 119-91.

4. Gulisashvili A. Analytically Tractable Stochastic Stock Price Models. Berlin: Springer (2012). doi: 10.1007/978-3-642-31214-4

5. Labordère PH. Analysis, Geometry, and Modeling in Finance: Advanced Methods in Option Pricing Boca Raton, FL: Chapman \& Hall (2009).

6. Rebonato R. Volatility and Correlation: The Perfect Hedger and the Fox. Chichester: John Wiley \& Sons (2004).

7. Heston SL. A closed-form solution for options with stochastic volatility with applications to bond and currency options. Rev Financ Stud. (1993) 6:327-43. doi: $10.1093 / \mathrm{rfs} / 6.2 .327$

8. Gatheral J, Jaisson T, Rosenbaum M. Volatility is rough. Quant Financ. (2018) 18:933-49. doi: 10.1080/14697688.2017.1393551

9. Cont R. Empirical properties of asset returns: stylized facts and statistical issues. Quant Finance. (2001) 1:1-14. doi: 10.1080/713665670

10. Black F. Studies in stock price volatility changes. In: Proceedings of the 1976 Business Meeting of the Business and Economic Statistics Section. American Statistical Association (1976). p. 177-81.

11. Mendes RV, Oliveira MJ, Rodrigues AM. The fractional volatility model: no-arbitrage, leverage and completeness. Phys A. (2015) 419:470-8. doi: $10.1016 /$ j.physa.2014.10.056

12. Garnier J, Sølna K. Correction to Black-Scholes formula due to fractional stochastic volatility. SIAM J Math Financ. (2017) 8:560-88. doi: $10.1137 / 15 \mathrm{M} 1036749$

13. Garnier J, Sølna K. Option pricing under fast-varying and rough stochastic volatility. Ann Finance. (2018) 14:489-516. doi: 10.1007/s10436-018-0325-4

14. Garnier J, Sølna K. Option pricing under fast-varying long-memory stochastic volatility. Math Finance. (2019) 29:39-83. doi: 10.1111/mafi.12186

15. Fouque J-P, Hu R. Optimal portfolio under fractional stochastic environment. Math Finance. (2019) 29:697-734. doi: 10.1111/mafi.12195

16. Fouque J-P, Hu R. Optimal portfolio under fast mean-reverting fractional stochastic environment. SIAM J Finan Math. (2018b) 6:564-601. doi: $10.1137 / 17 \mathrm{M} 1134068$

17. Fouque J-P, Hu R. Portfolio optimization under fast mean-reverting and rough fractional stochastic environment. Appl Math Finance. (2018a) 25:361-88. doi: 10.1080/1350486X.2019.1584532

18. Garnier J, Sølna K. Optimal hedging under fast-varying stochastic volatility. SIAM J Math Financ. (2020). doi: 10.1137/18M1221655

19. Aït-Sahalia Y, Fan J, Li J. Testing for jumps in a discretely observed process. Ann Stat. (2009) 37:184-222. doi: 10.1214/07-AOS568

20. Carr P, Wu L. What type of processes underlies options? A simple robust test. J Finance. (2003) 58:2581-610. doi: 10.1046/j.1540-6261.2003.00616.x modeling with fractional short- and long-range processes, for instance to hedging as in Garnier and Sølna [18] and to optimal portfolio construction as in Fouque and $\mathrm{Hu}$ [15-17]. It is also possible to discuss other asymptotic regimes with small volatility fluctuations as in Garnier and Sølna [12] and short time to maturity as in Alòs et al. [39].

\section{AUTHOR CONTRIBUTIONS}

The research has been been carried out by JG and KS. Both authors have written and agreed on the final manuscript.

21. Figueroa-López JE, Olafsson S. Short-time expansions for close-to-the-money options under a Lévy jump model with stochastic volatility. arXiv:1404.0601 (2014). doi: 10.1007/s00780-015-0281-z

22. Mijatovic A, Tankov P. A new look at short-term implied volatility in asset price models with jumps. Math Financ. (2016) 26:149-83. doi: $10.1111 /$ mafi. 12055

23. Barndorff-Nielsen OE, Benth FE, Veraart AED. Modelling energy spot prices by volatility modulated Lévy driven Volterra processes. Bernoulli. (2013) 19:80-845. doi: 10.3150/12-BEJ476

24. Breidt FJ, Crato N, De Lima P. The detection and estimation of long-memory in stochastic volatility. J Econ. (1998) 83:325-48. doi: 10.1016/S0304-4076(97)00072-9

25. Charfeddine L. True or spurious long memory in volatility: further evidence on the energy futures markets. Energy Policy. (2014) 71:76-93. doi: 10.1016/j.enpol.2014.04.027

26. Chronopoulou A, Viens FG. Estimation and pricing under longmemory stochastic volatility. Ann Finance. (2012a) 8:379-403. doi: 10.1007/s10436-010-0156-4

27. Oh G, Kim S, Eom C. Long-term memory and volatility clustering in high-frequency price changes. Phys A. (2008) 387:1247-54. doi: 10.1016/j.physa.2007.08.061

28. Mandelbrot BB, Van Ness JW. Fractional Brownian motions, fractional noises and applications. SIAM Rev. (1968) 10:422-37. doi: 10.1137/1010093

29. Biagini F, Hu Y, Øksendal B, Zhang T. Stochastic Calculus for Fractional Brownian Motion and Applications. London: Springer (2008).

30. Comte F, Coutin L, Renault E. Affine fractional stochastic volatility models. Ann Finance. (2012) 8:337-78. doi: 10.1007/s10436-010-0165-3

31. Comte F, Renault E. Long memory in continuous-time stochastic volatility models. Math Finance. (1998) 8:291-323. doi: 10.1111/1467-9965.00057

32. El Euch O, Rosenbaum M. Perfect hedging in rough Heston models. Ann Appl Probab. (2018) 28:3813-56. doi: 10.1214/18-AAP1408

33. Guennoun H, Jacquier A, Roome P. Aymptotic behaviour of the fractional Heston model. arXiv:1411.7653 (2014). doi: 10.2139/ssrn.25 31468

34. Walther T, Klein T, Thu HP,Piontek K. True or spurious long memory in European non-EMU currencies. Res Int Bus Finance. (2017) 40:217-30. doi: 10.1016/j.ribaf.2017.01.003

35. Chia KC, Bahar A, Kane IL, Ting C-M, Rahman HA. Estimation of stochastic volatility with long memory for index prices of FTSE Bursa Malaysia KLCI. AIP Conference Proceedings (2015). p. 1643. doi: 10.1063/1.49 07427

36. Bennedsen M. Rough Electricity: A New Fractal Multi-Factor Model of Electricity Spot Prices. Working Paper (2015). Available online at: http://ssrn. com/abstract $=2636829$

37. Rypdal M, Løvsletten O. Modeling electricity spot prices using mean-reverting multifractal processes. Phys A. (2013) 392:194-207. doi: 10.1016/j.physa.2012.08.004

38. Simonsen I. Measuring anti-correlations in the nordic electricity spot market by wavelets. Phys A. (2002) 233:597-606. doi: 10.1016/S0378-4371(02)0 1938-6 
39. Alòs E, León JA, Vives J. On the short-time behavior of the implied volatility for jump-diffusion models with stochastic volatility. Finance Stoch. (2007) 11:571-89. doi: 10.1007/s00780-007-0049-1

40. Bates DS. Jumps and stochastic volatility: exchange rate processes implicit in Deutsche Mark options. Rev Finance Stud. (1996) 9:69-107. doi: 10.1093/rfs/9.1.69

41. Forde M, Zhang H. Asymptotics for Rough Stochastic Volatility and Lévy Models. Available online at: http://www.mth.kcl.ac.uk/fordem/

42. Chronopoulou A, Viens FG. Stochastic volatility models with long-memory in discrete and continuous time. Quant Finance. (2012b) 12:635-49. doi: 10.1080/14697688.2012.664939

43. Gulisashvili A, Viens F, Zhang X. Small-time asymptotics for Gaussian self-similar stochastic volatility models. Appl Math Optim. (2015). doi: 10.1007/s00245-018-9497-6

44. Fukasawa M. Asymptotic analysis for stochastic volatility: martingale expansion. Financ Stoch. (2011) 15:635-54. doi: 10.1007/s00780-010-0 136-6
45. Corley S, Lebovits J, Lévy Véhel J. Multifractional stochastic volatility models. Math Finance. (2014) 24:364-402. doi: 10.1111/mafi.12024

46. Fouque J-P, Papanicolaou G, Sircar KR. Derivatives in Financial Markets with Stochastic Volatility. Cambridge: Cambridge University Press (2000).

47. Fouque J-P, Papanicolaou G, Sircar KR, Sølna K. Maturity cycles in implied volatility. Financ Stochast. (2004) 8:451-77. doi: 10.1007/s00780-004-0126-7

Conflict of Interest: The authors declare that the research was conducted in the absence of any commercial or financial relationships that could be construed as a potential conflict of interest.

Copyright (c) 2020 Garnier and Sølna. This is an open-access article distributed under the terms of the Creative Commons Attribution License (CC BY). The use, distribution or reproduction in other forums is permitted, provided the original author(s) and the copyright owner(s) are credited and that the original publication in this journal is cited, in accordance with accepted academic practice. No use, distribution or reproduction is permitted which does not comply with these terms. 